\title{
Emergence of sprite streamers from screening-ionization waves in the lower ionosphere
}

\author{
Alejandro Luque ${ }^{1 \star}$ and Ute Ebert ${ }^{1,2 \star}$
}

Sprite discharges above thunderclouds at altitudes of 40-90 km (refs 1-5) are usually created by a strong positive cloud-to-ground lightning flash ${ }^{6}$. Sometimes these sprite discharges emerge from a visible halo ${ }^{5,7-9}$, and during the first stage they always propagate downwards and branch on their way, 5,9-11. Modelling efforts have been restricted to conditions of non-ionized air of constant density and show double-headed sprites $^{12}$ or sprites starting from metal electrodes, but they do not explain why observations exclusively record sprites that propagate downwards. Here we present simulations with a numerical discharge model on a non-uniform, dynamically adapted computational grid $^{13}$ to capture the wide range of emerging spatial scales, and we use realistic air and electron densities that vary with altitude. Our model shows a downward-propagating screening-ionization wave in the lower ionosphere that sharpens and collapses into a sprite streamer as it propagates farther down. Streamer velocity, diameter and length until branching agree with observations ${ }^{9}$ within measuring accuracy. We speculate that sprites generically emerge through the collapse of a wide screening-ionization wave into a sprite streamer, although this wave is only sometimes visible as a luminous halo.

Electrical breakdown in the upper atmosphere above a thundercloud was predicted ${ }^{1}$ in 1925 . However, only in 1990 was an 'unusual luminous electrical discharge' actually reported ${ }^{2}$ in the scientific literature. The most frequent transient luminous events $^{4}$ are elves ${ }^{7}$ and sprites ${ }^{14}$. Sprites are strongly luminous ${ }^{11}$, filamentary ${ }^{15}$ discharges in the mesosphere. Continued progress in instrumentation has uncovered their complex dynamics in ever increasing temporal ${ }^{9,16}$, spatial ${ }^{15}$ and spectral $^{7,14}$ resolution. Sprites start as downward-propagating filaments, often emerging from a diffuse glow, called a halo ${ }^{7-9,14}$. Only after these initial discharges have propagated about $10 \mathrm{~km}$ down within milliseconds, a new phase of upward-propagating discharges is observed ${ }^{5}$.

After estimates of electric breakdown high above thunderclouds more than 80 years ago $^{1}$, sprite theory was largely shaped in the past 13 years ${ }^{17,18}$. The thin filaments of sprites are streamers ${ }^{19}$ : thin plasma channels that propagate into a non-ionized medium exposed to a high electric field. The streamer nature of sprites was confirmed by telescopic imaging ${ }^{15}$ and by movies with submillisecond time steps ${ }^{5,11,16}$. As lengths and times for streamers scale with air density, small streamers at atmospheric pressure and large sprites at high altitudes ${ }^{5,16,20}$ are physically similar ${ }^{12,20,21}$. Streamer models have been used ${ }^{12}$ to study sprites, but only over short propagation lengths where the variation of air density can be neglected. Also background ionization was neglected. Rather the streamers were initiated by arbitrary localized ionization seeds, creating either double-headed streamers extending at both ends simultaneously, or streamers were launched from a pointed metal electrode. However, such sprite streamers were never observed: upward streamers emerge from the body of an earlier downward streamer ${ }^{5}$. Moreover, those models did not reach the measured sprite velocities of $10^{7} \mathrm{~m} \mathrm{~s}^{-1}$ and diameters of hundreds of metres.

Modelling the emergence of sprites from the ionosphere was first attempted in ref. 22, but the low accuracy of observations and numerical models available at the time led the authors to misinterpret the wave of about $15 \mathrm{~km}$ width that starts in the ionosphere as a sprite or sprite 'hair', whereas today we would call it the initial halo. Later evolution stages could not be followed.

Here we use up-to-date numerical tools with adaptive grid refinement, realistic altitude-dependent air and electron density profiles and a simple model of the electric field evolution above a positive cloud-to-ground lightning stroke. We study the inception of streamers from haloes and observe a novel physical phenomenon: the collapse of a wide screening-ionization wave and the sudden transition into the sprite streamer mode of propagation.

Our discharge model, detailed in the Supplementary Information, contains electron drift and diffusion, and the dynamically most relevant reactions: impact ionization, electron attachment and photo-ionization ${ }^{23}$. Light emission from impact excitation is included as in ref. 12. Transport and reaction rates depend on local air density and on the local reduced electric field $E / E_{\mathrm{k}}$, where $E$ is the electric field, $E_{\mathrm{k}} \approx 32 \mathrm{kV} \mathrm{cm}{ }^{-1} \cdot N / N_{0}$ is the breakdown field and $N$ and $N_{0}$ are the air densities at a given altitude and at sea level. The model is standard for streamers; however, current halo models ${ }^{17,24-26}$ include electric currents but conductivity is not treated self-consistently (see Supplementary Information).

A streamer discharge has an intricate inner structure whereas it occupies only a tiny fraction of the total simulation volume; therefore, a non-uniform dynamically adapted numerical grid ${ }^{13,23}$ must be used as demonstrated for atmospheric pressure streamers ${ }^{21,27}$. Our present simulations follow electron and ion densities over altitudes from 55 to $85 \mathrm{~km}$ and over a radius of $20 \mathrm{~km}$; the finest grid used locally is $3 \mathrm{~m}$.

In our model, the air density $N(z) \sim \mathrm{e}^{-z / h}$ decays with altitude $z$ over the length $h=7.2 \mathrm{~km}$. The night-time electron density $n_{\mathrm{e}}(z) \sim \mathrm{e}^{z / \ell}$ due to cosmic radiation is modelled as exponentially increasing with altitude with a characteristic length $\ell=2.9 \mathrm{~km}$ (ref. 25). Ionic conductivity is significantly smaller than electron conductivity above $60 \mathrm{~km}$ altitude ${ }^{17}$ and neglected.

For the lightning stroke, we use a standard model ${ }^{28}$ for a shortdelayed sprite assuming that the cloud is initially charge neutral, and that the stroke moves positive charge to the ground with a current of $30 \mathrm{kA}$. The fields are then approximated by assuming that a negative point charge builds up at $L=10 \mathrm{~km}$ altitude. $(75 \mathrm{kA}$ from $4 \mathrm{~km}$ altitude generates the same fields.) As detailed later, a sprite emerges after $\sim 4.3 \mathrm{~ms}$, when the charge moment change is approximately $L Q=1,300 \mathrm{Ckm}$, known to initiate sprites with a probability of

${ }^{1}$ Centrum voor Wiskunde en Informatica (CWI), PO Box 94079, 1090 GB Amsterdam, The Netherlands, ${ }^{2}$ Department of Applied Physics, Eindhoven University of Technology, The Netherlands. *e-mail: luque@cwi.nl; ebert@cwi.nl. 

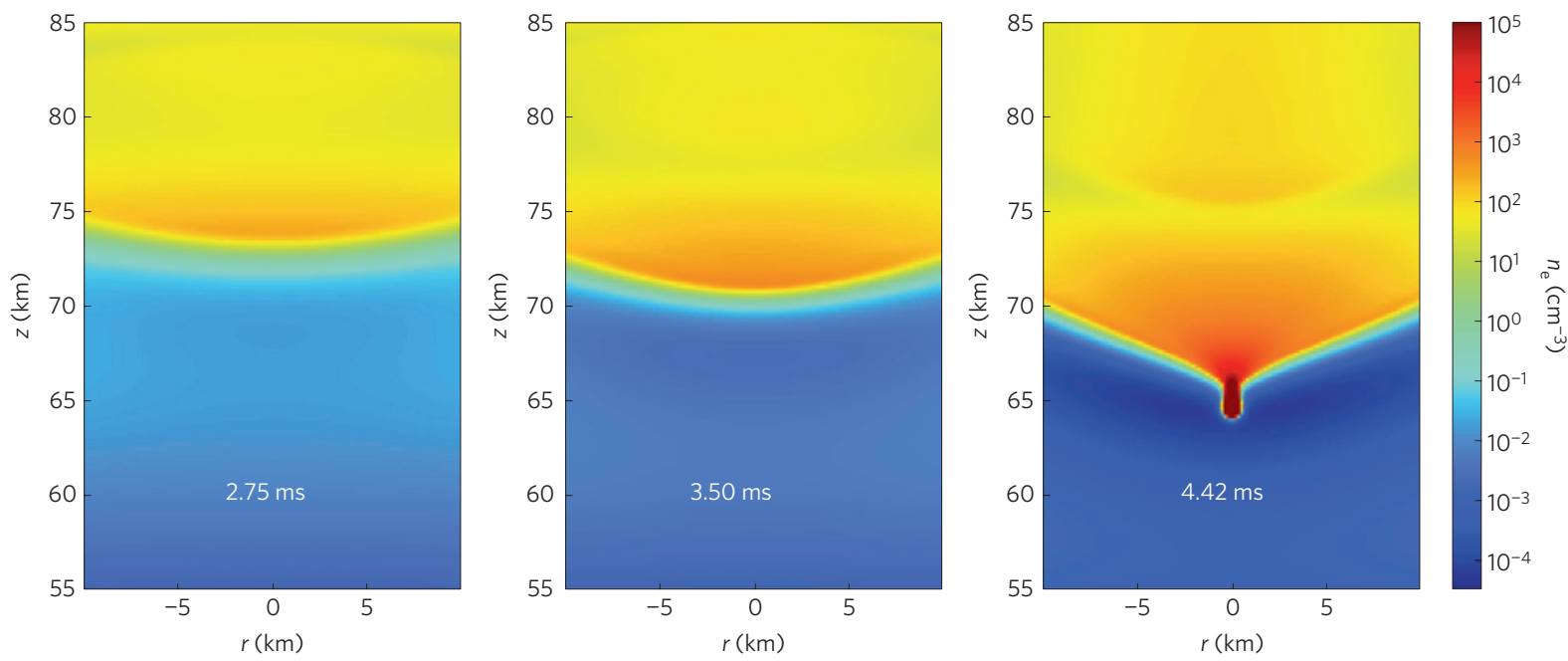

Figure 1 | The simulated electron density develops from sprite halo to primary sprite streamer. The electron density $n_{\mathrm{e}}$ on a logarithmic scale at times $2.75,3.50$ and $4.42 \mathrm{~ms}$ while a positive lightning stroke creates a charge moment at a rate of $300 \mathrm{Ckm} \mathrm{ms}^{-1}$. The relaxation-ionization wave becomes sharper, and a sprite streamer emerges right above the thundercloud charge. Secondary ionization waves emerge above the main one, but they hardly move. See also the Supplementary Information for the reduced electric field, for zooms into the sprite and for movies of the full temporal evolution.
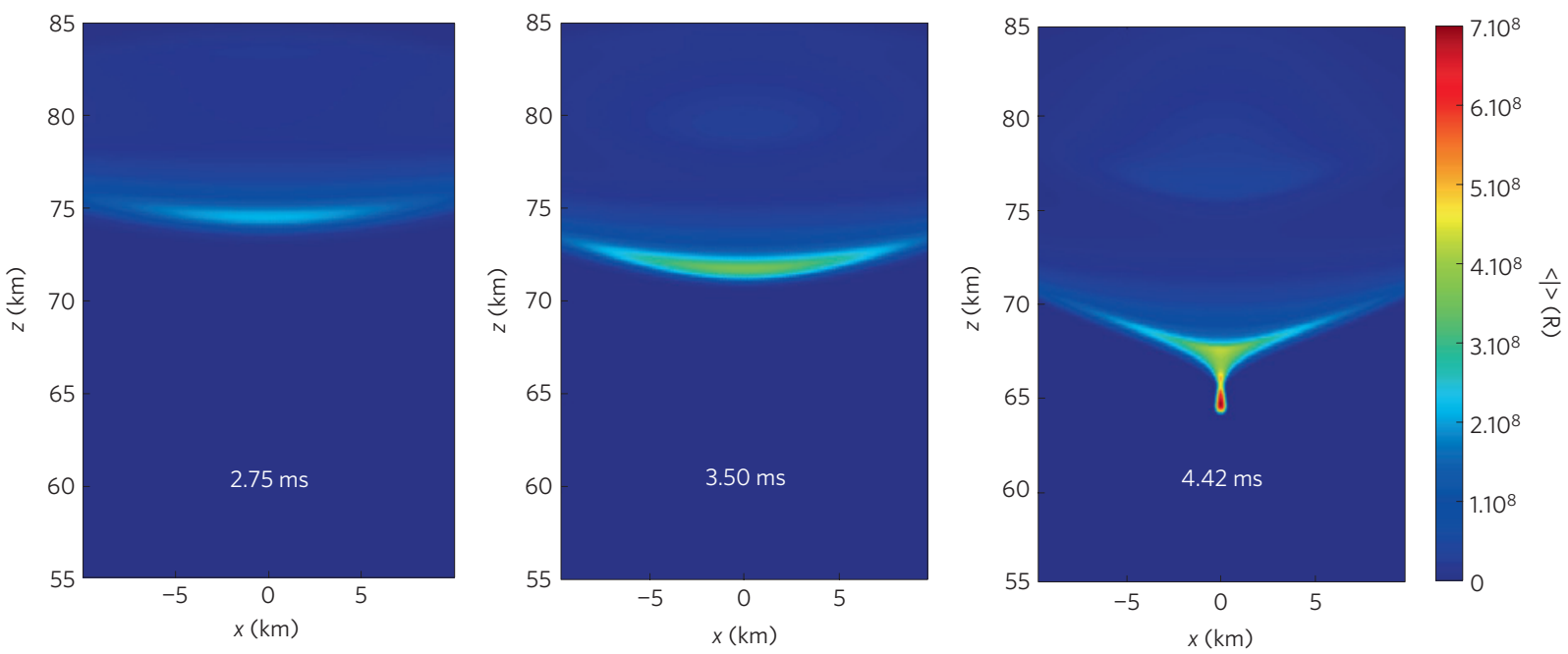

Figure 2 | Optical observations show a faint halo followed by a downward-propagating bright filament. The predicted optical emissions in the first positive band of molecular nitrogen, averaged over $0.25 \mathrm{~ms}$ and integrated over a line of sight perpendicular to the plane of the figure. The intensity is given in rayleigh ( $\mathrm{R}$ ); details are given in the Supplementary Information.

over $90 \%$ (refs 2, 6). (Sprite emergence also depends on lightning current and duration ${ }^{14,17,24-26}$.) As electrical conductivity largely increases with altitude, we model the Earth and the ionosphere above $85 \mathrm{~km}$ as perfectly conducting and solve the electrostatic Poisson equation over the whole altitude range from 0 to $85 \mathrm{~km}$ and up to a radius of $20 \mathrm{~km}$.

We emphasize that in our model no artificial initial ionization seeds need to be placed. Figure 1 shows relevant stages of the simulated electron density and Fig. 2 the corresponding light emission. Out of an initially exponentially increasing electron density profile, an increasingly sharp wavefront emerges that we call a screening-ionization wave. Eventually it creates an electron density shock wave from which a downward-propagating sprite streamer emerges $4.3 \mathrm{~ms}$ after the start of the lightning stroke. The diameter of enhanced electron density in the sprite streamer is $\sim 1 \mathrm{~km}$; as calculated in the Supplementary Information, this corresponds to an optical diameter of $\sim 600 \mathrm{~m}$. The streamer velocity is $3 \times 10^{7} \mathrm{~m} \mathrm{~s}^{-1}$. The evolution strongly resembles highspeed recordings ${ }^{9}$, where a sprite streamer of 300-400 $\mathrm{m}$ diameter emerges $2 \mathrm{~ms}$ after the start of the parent lightning (return) stroke from a large (about $20 \mathrm{~km}$ wide) halo and propagates downwards with a velocity of $\sim 10^{7} \mathrm{~m} \mathrm{~s}^{-1}$. In our simulation, the streamer branches into many channels after propagating $\sim 2 \mathrm{~km}$ as a single channel, similarly to the observations ${ }^{9}$. The streamer diameter is comparable to the widest streamers observed telescopically ${ }^{15}$. Recent laboratory experiments ${ }^{20,29}$ have classified streamers of different diameters and their properties ${ }^{20}$, and have confirmed the quantitative extrapolation ${ }^{29}$ to sprites. As our initial sprite streamer branches heavily after a short propagation distance, it must be of type 1 in the terminology of ref. 29 or even wider, that is, at least one or two orders of magnitude wider than a minimal streamer at the same air density.

The underlying dynamics are illustrated in more detail in Fig. 3. It shows the evolution of the reduced electric field $E / E_{\mathrm{k}}$ and of the electron density $n_{\mathrm{e}}$ (on a logarithmic scale) within a number of time steps on the vertical axis through cloud charge, halo and sprite. The field $E$ that builds up by the lightning stroke varies little in space as long as the air is electrically neutral; therefore, 

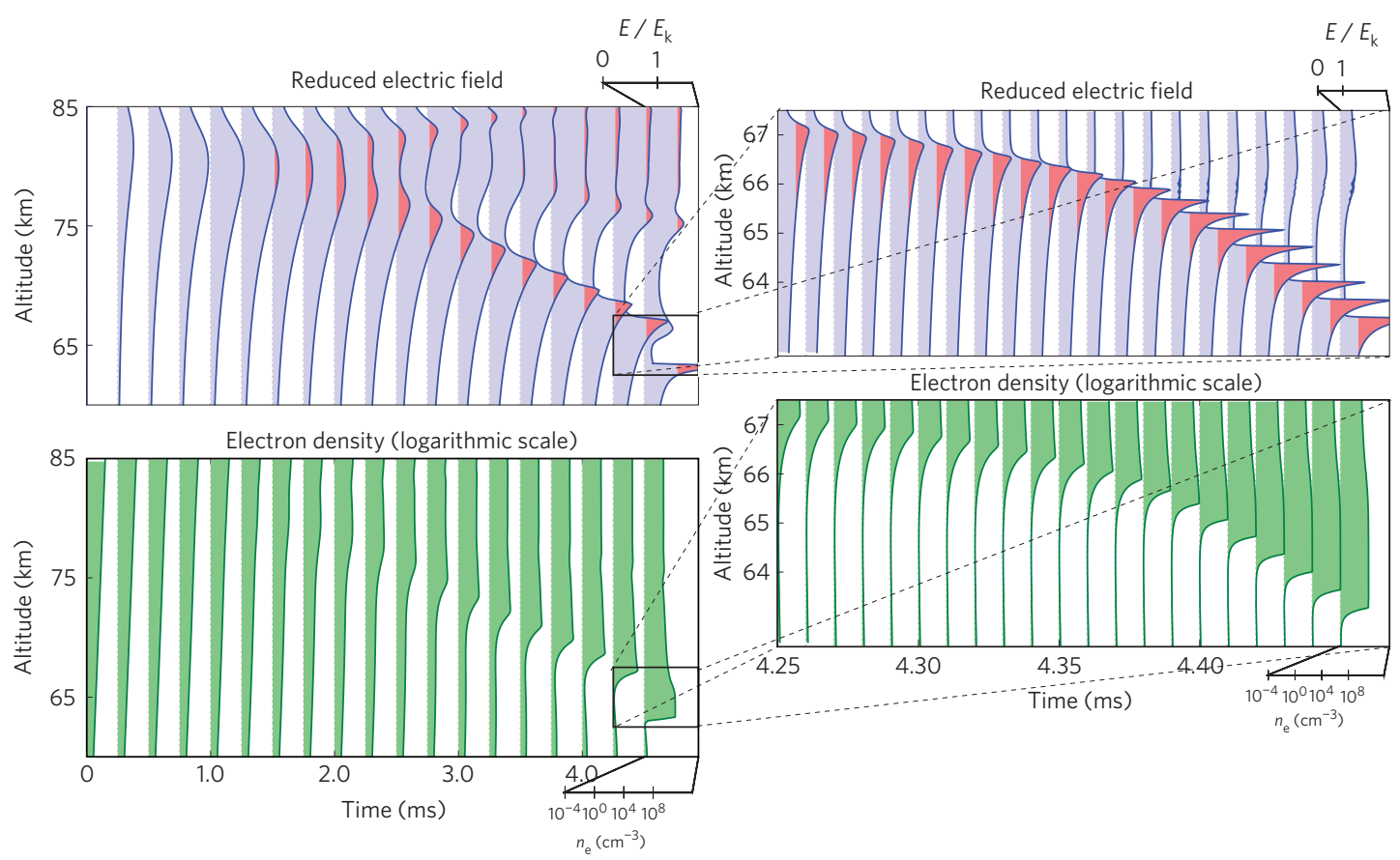

Figure 3 | The reduced electric field $E / E_{k}$ and the electron density $n_{e}$ develop on the axis of the simulation. Movies of the full evolution of both quantities are included in the Supplementary Information. The regions where impact ionization dominates over attachment $\left(E / E_{k}>1\right)$ are shaded in red in the field panels. The left panels show the evolution of the complete simulation time and altitude up to the time of collapse of $4.3 \mathrm{~ms}$; time steps are $0.25 \mathrm{~ms}$. The right panels zoom, both in time and in space, into the ionization wave collapse and the emergence of the sprite streamers.

the reduced electric field $E / E_{\mathrm{k}}$ increases exponentially with altitude. As conductivity increases exponentially as well, higher altitudes get electrically screened earlier by upward motion of the electrons, and an electrical screening wave of remaining positive charge moves downwards ${ }^{17}$. On the leading edge of that wave, the electric field exceeds $E_{\mathrm{k}}$ after about $1.25 \mathrm{~ms}$ and the electrons multiply, feeding the further propagation of the wave. This screening-ionization wave propagates mainly owing to high background electron density. The typical length scale in this stage is hence the decay length of the background ionization, $\sim 2.9 \mathrm{~km}$.

Below the edge of the wave, owing to increasing air density, $E_{\mathrm{k}}$ increases faster than $E$, and electron attachment dominates over impact ionization; therefore, electron density and conductivity decrease in time, owing to field-induced attachment. Furthermore, the electrons in this region keep drifting upwards, against the advancing screening-ionization wave. Finally, as propagation is slower at lower altitudes owing to higher gas densities, any structure that moves downwards is compressed. The combination of these three processes steepens the wavefront, which eventually collapses at time $t \approx 4.3 \mathrm{~ms}$. At that moment, the electron density below the front is so low (about $10^{-4} \mathrm{~cm}^{-3}$ ) that the propagation by background ionization is not sustained anymore. The electric field then focuses strongly and the wave enters the streamer mode with strong field enhancement at the tip and propagation by photo-ionization. The dominant length scale hence turns from the decay length of the background ionization to the much smaller length scale of photo-ionization (about $12 \mathrm{~m}$ at $67 \mathrm{~km}$ altitude). The fine structures inside the streamer can be treated only by adaptive grid refinement ${ }^{13}$.

Previous theory ${ }^{12,18}$ of streamer propagation and branching at sprite altitudes included photo-ionization and three-body processes, but neglected background ionization. Our results show that this assumption is valid once the streamer phase is reached. However, the earlier stage of the screening-ionization wave strongly depends on the background ionization. Unfortunately, measuring those profiles during a thunderstorm is difficult, so we relied on estimates based on normal conditions during the night time. However, it has been argued ${ }^{30}$ that the electric activity of the thunderstorm changes the electron density above the thundercloud into density profiles from which ionization waves and sprite streamers can emerge more easily; and it was proposed ${ }^{7}$ to use optical observations. By presenting a new mechanism of sprite initiation, our calculations may furthe $r$ help to estimate the ionization profiles during a thunderstorm. Counter-intuitively, an increased atmospheric conductivity may prevent the appearance of sprites. When we run simulations with an initial ionization five times higher than our base calculations, the front of the initial wave was much smoother and the charge moment change required to initiate a sprite was $\sim 1,450 \mathrm{Ckm}$.

The recent Imager for Sprites and Upper Atmospheric Lightning satellite data ${ }^{14}$ reports a charge moment change of $\sim 1,300 \mathrm{Ckm}$ for sprite formation as an average over more than 300 events, in quantitative agreement with our results. However, sprites have been seen to be generated by charge moment changes as low as $\sim 500 \mathrm{Ckm}$, although the probability of such events is under $10 \%$ (refs 9, 24). In our simulations, such events produce electric fields above the ionization threshold at high altitudes, confirming the results of ref. 25 . Then a wide ionization wave propagates downwards and a steep gradient in the electron density is created but no streamer emerges from it, in agreement with most observations. Probably a horizontally inhomogeneous electron or air density is needed to destabilize the collapsed ionization front and create a sprite. We have studied this effect in simulations with higher initial electron density on the axis. The results, presented in the Supplementary Information, show that sprites emerge significantly earlier, higher and with lower charge moment changes. A stronger inhomogeneity can be simulated by a small bump in the electron density close to the screening-ionization wavefront. That would initiate a streamer after a flash of $500 \mathrm{Ckm}$. The need for significant inhomogeneities would explain the low probability of those observations. 
We have presented a mechanism that explains the observed destabilization of a halo and the emergence of sprites. However, not all streamers observed in sprites emerge from a visible halo and haloes may exist also without volume ionization ${ }^{26}$. Following ref. 7, we speculate that in many cases sprites emerge from wave collapses but that light emissions from the wave are not intense enough to be detected as a halo. This would explain the absence of upward-propagating streamers in the early stages of sprites. This is a matter of future modelling and observations.

Received 29 June 2009; accepted 23 September 2009; published online 25 October 2009

\section{References}

1. Wilson, C. T. R. The electric field of a thundercloud and some of its effects. Proc. Phys. Soc. Lond. 37, 32D-37D (1925).

2. Franz, R. C., Nemzek, R. J. \& Winckler, J. R. Television image of a large upward electrical discharge above a thunderstorm system. Science 249, 48-51 (1990).

3. Sentman, D. D., Wescott, E. M., Osborne, D. L., Hampton, D. L. \& Heavner, M. J. Preliminary results from the Sprites 94 aircraft campaign: 1. Red sprites. Geophys. Res. Lett. 22, 1205-1208 (1995).

4. Neubert, T. et al. Recent results from studies of electric discharges in the mesosphere. Surv. Geophys. 14, 71-137 (2008).

5. Stenbaek-Nielsen, H. C. \& McHarg, M. G. High time-resolution sprite imaging: Observations and implications. J. Phys. D 41, 234009 (2008).

6. Huang, E. et al. Criteria for sprites and elves based on Schumann resonance observations. J. Geophys. Res. 104, 16943-16964 (1999).

7. Barrington-Leigh, C. P., Inan, U. S. \& Stanley, M. Identification of sprites and elves with intensified video and broadband array photometry. J. Geophys. Res. 106, 1741-1750 (2001).

8. Pasko, V. P. \& Stenbaek-Nielsen, H. C. Diffuse and streamer regions of sprites Geophys. Res. Lett. 29, 1440 (2002).

9. Cummer, S. A. et al. Submillisecond imaging of sprite development and structure. Geophys. Res. Lett. 33, L04104 (2006).

10. Stanley, M. et al. High speed video of initial sprite development. Geophys. Res. Lett. 26, 3201-3204 (1999).

11. Stenbaek-Nielsen, H. C., McHarg, M. G., Kanmae, T. \& Sentman, D. D. Observed emission rates in sprite streamer heads. Geophys. Res. Lett. 34, L11105 (2007)

12. Liu, N. \& Pasko, V. P. Effects of photoionization on propagation and branching of positive and negative streamers in sprites. J. Geophys. Res. 109, A04301 (2004).

13. Montijn, C., Hundsdorfer, W. \& Ebert, U. An adaptive grid refinement strategy for the simulation of negative streamers. J. Comput. Phys. 219, 801-835 (2006).

14. Adachi, T. et al. Electric fields and electron energies in sprites and temporal evolutions of lightning charge moment. J. Phys. D 41, 234010 (2008).

15. Gerken, E. A., Inan, U. S. \& Barrington-Leigh, C. P. Telescopic imaging of sprites. Geophys. Res. Lett. 27, 2637-2640 (2000).

16. McHarg, M. G., Stenbaek-Nielsen, H. C. \& Kammae, T. Observations of streamer formation in sprites. Geophys. Res. Lett. 34, L06804 (2007).
17. Pasko, V. P., Inan, U. S., Bell, T. F. \& Taranenko, Y. N. Sprites produced by quasi-electrostatic heating and ionization in the lower ionosphere. J. Geophys. Res. 102, 4529-4561 (1997).

18. Pasko, V. P. Red sprite discharges in the atmosphere at high altitude: The molecular physics and the similarity with laboratory discharges. Plasma Sources Sci. Technol. 16, S13-S29 (2007).

19. Ebert, U. et al. The multiscale nature of streamers. Plasma Sources Sci. Technol. 15, S118-S129 (2006).

20. Briels, T. M. P., van Veldhuizen, E. M. \& Ebert, U. Positive streamers in air and nitrogen of varying density: Experiments on similarity laws. J. Phys. D 41, 234008 (2008)

21. Luque, A., Ebert, U. \& Hundsdorfer, W. Interaction of streamer discharges in air and other oxygen-nitrogen mixtures. Phys. Rev. Lett. 101, 075005 (2008).

22. Pasko, V. P., Inan, U. S. \& Bell, T. F. Sprites as luminous columns of ionization produced by quasi-electrostatic thundercloud fields. Geophys. Res. Lett. 23, 649-652 (1996).

23. Luque, A., Ebert, U., Montijn, C. \& Hundsdorfer, W. Photoionization in negative streamers: Fast computations and two propagation modes. Appl. Phys. Lett. 90, 081501 (2007).

24. Hu, W., Cummer, S. A., Lyons, W. A. \& Nelson, T. E. Lightning charge moment changes for the initiation of sprites. Geophys. Res. Lett. 29, 1279 (2002).

25. Hu, W., Cummer, S. A. \& Lyons, W. A. Testing sprite initiation theory using lightning measurements and modelled electromagnetic fields. J. Geophys. Res. 112, D13115 (2007)

26. Hiraki, Y. \& Fukunishi, H. Theoretical criterion of charge moment change by lightning for initiation of sprites. J. Geophys. Res. 111, A11305 (2007).

27. Luque, A., Ratushnaya, V. \& Ebert, U. Positive and negative streamers in ambient air: Modelling evolution and velocities. J. Phys. D 41, 234005 (2008).

28. Raizer, Y. P., Milikh, G. M., Shneider, M. N. \& Novakovski, S. V. Long streamers in the upper atmosphere above thundercloud. J. Phys. D 31, 3255-3264 (1998).

29. Briels, T. M. P., Kos, J., van Veldhuizen, E. M. \& Ebert, U. Circuit dependence of the diameter of pulsed positive streamers in air. J. Phys. D 39, 234008 (2006).

30. Marshall, R. A., Inan, U. S. \& Chevalier, T. W. Early VLF perturbations caused by lightning EMP-driven dissociative attachment. Geophys. Res. Lett. 35, L21807 (2008).

\section{Acknowledgements}

A.L. acknowledges financial support by STW-projects 06501 and 11018 of The Netherlands' Organization for Scientific Research (NWO). We thank E. Williams for valuable remarks and suggestions.

\section{Author contributions}

All simulations were carried out by A.L., based on numerical methods developed earlier by C. Montijn et al. (ref. 13) and on his own further developments ${ }^{21,23}$. The problem of sprite streamer propagation in varying air density was posed by U.E.; A.L. included the electron density variation when approaching the ionosphere, and hence effectively the halo part. Evaluations, interpretations and literature studies were carried out together.

\section{Additional information}

Supplementary information accompanies this paper on www.nature.com/naturegeoscience. Reprints and permissions information is available online at http://npg.nature.com/ reprintsandpermissions. Correspondence and requests for materials should be addressed to A.L. or U.E. 


\title{
Emergence of sprite streamers
}

\section{from screening-ionization waves in the lower ionosphere}

\author{
Alejandro Luque*, Ute Ebert ${ }^{1,2}$ \\ ${ }^{1}$ Centrum voor Wiskunde en Informatica (CWI), \\ P.O. Box 94079, 1090 GB Amsterdam, \\ The Netherlands, \\ email: luque@cwi.nl, ebert@cwi.nl, \\ +31-20-592 4206 (tel Ebert), 4199 (fax) and \\ ${ }^{2}$ Dept. Appl. Physics, Eindhoven Univ. Techn., The Netherlands \\ (Dated: July 23, 2009)
}




\section{SUPPLEMENTARY INFORMATION}

We use the discharge model described in [19] and extend it by electron attachment to oxygen and by altitude-dependent transport and ionization parameters:

$$
\begin{aligned}
\partial_{t} n_{e}= & \nabla \cdot\left(n_{e} \mu(N) \mathbf{E}\right)+\nabla \cdot\left(D(N) \nabla n_{e}\right) \\
& +\left(v_{i}-v_{a t t}\right) n_{e}+S_{p h}, \\
\partial_{t} n_{+}= & v_{i} n_{e}+S_{p h}, \\
\partial_{t} n_{-}= & v_{a t t} n_{e}, \\
v_{i}= & \mu(N)|\mathbf{E}| \alpha_{i}(N) e^{-E_{i}(N) /|\mathbf{E}|}, \\
v_{\text {att }}= & \mu(N)|\mathbf{E}| \alpha_{a t t}(N) e^{-E_{a t t}(N) /|\mathbf{E}|}, \\
\epsilon_{0} \nabla \cdot \mathbf{E}= & \mathrm{e}\left(n_{+}-n_{-}\right), \quad \mathbf{E}=-\nabla \phi .
\end{aligned}
$$

Here $N$ is the altitude dependent number density of neutral air molecules, $\mu(N)$ is the electron mobility, $D(N)$ is the electron diffusion, $\alpha_{i}(N)$ and $\alpha_{a t t}(N)$ are the inverse of the mean free paths of electrons between ionization or attachment events, respectively, and e is the elementary charge. Ion mobility, much smaller than electron mobility, is neglected. The term $S_{p h}$ stands for the nonlocal photo-ionization according to the standard model for oxygen-nitrogen mixtures [23]. In its standard formulation that model assumes a homogeneous density of absorbing $\mathrm{O}_{2}$ molecules and hence an isotropic absorption function. This assumption does not hold for sprites. However, we use the following approximation:

$$
S_{p h}(\mathbf{r})=\frac{\xi A(N(Z))}{4 \pi} \int \frac{h\left(N(Z)\left|\mathbf{r}-\mathbf{r}^{\prime}\right|\right) S_{i}\left(\mathbf{r}^{\prime}\right) d^{3}\left(p \mathbf{r}^{\prime}\right)}{\left|N(Z) \mathbf{r}-N(Z) \mathbf{r}^{\prime}\right|^{2}},
$$

where $S_{i}=v_{i} n_{e}, A(N)$ is a quenching factor, $h$ is the (isotropic) absorption function of ionizing radiation [23] and $Z$ is the $z$-coordinate of the maximum of $v_{i} n_{e}$. Here we are assuming that photoionization is relevant only close to the tip of a propagating front, where the impact ionization is highest. This approximation is justified by the result presented in the main text of the article: during a first stage of propagation, photo-ionization plays a minor and mostly local role. Later it becomes important for the propagation of thin streamers but their diameter as well as the photoionization length are then much smaller than the decay length of $N(z)$.

The number density at a given altitude $z$ is taken as $N=N_{0} \exp (-z / h)$, where $h=7.2 \mathrm{~km}$ and $N_{0}=2.5 \cdot 10^{19} \mathrm{~cm}^{-3}$ is the air density at ground level. The dependence of equations (1)-(5) on the neutral density is detailed in [19]; it is $\mu(N)=N_{0} \mu_{0} / N, D(N)=N_{0} D_{0} / N, \alpha_{i}(N)=N \alpha_{i 0} / N_{0}$, 


\begin{tabular}{lcl}
\hline Parameter & Value at ground level & Scaling factor \\
\hline Electron mobility & $\mu_{0}=380 \mathrm{~cm}^{2} \mathrm{~V}^{-1} \mathrm{~s}^{-1}$ & $N_{0} / N$ \\
Electron diffusion rate & $D_{0}=1800 \mathrm{~cm}^{2} \mathrm{~s}^{-1}$ & $N_{0} / N$ \\
Townsend ionization rate & $\alpha_{i 0}=4332 \mathrm{~cm}^{-1}$ & $N / N_{0}$ \\
Townsend ionization field & $E_{i 0}=2 \cdot 10^{5} \mathrm{~V} \mathrm{~cm}^{-1}$ & $N / N_{0}$ \\
Townsend attachment rate & $\alpha_{a t t 0}=20 \mathrm{~cm}^{-1}$ & $N / N_{0}$ \\
Townsend attachment field & $E_{\text {att } 0}=3 \cdot 10^{4} \mathrm{~V} \mathrm{~cm}^{-1}$ & $N / N_{0}$ \\
\hline
\end{tabular}

TABLE I: Parameters of our model, with their values at ground level and how they scale with the air molecule number density $N$. Here $N_{0}$ is the air molecule number density at ground level.

$\alpha_{\text {att }}(N)=N \alpha_{\text {att } 0} / N_{0}, E_{i}(N)=N E_{i 0} / N_{0}, E_{\text {att }}(N)=N \alpha_{\text {att } 0} / N_{0}, A(N)=N_{q} /\left(N+N_{q}\right)$ with $N_{q} \approx$ $0.08 N_{0}$ [12] where $X_{0}$ indicates the value of $X$ at sea level, taken as in [19]. A summary of the model parameters and their values at ground level is provided in Table I.

The dependence on the air density of the impact ionization and attachment rates becomes clearer by noticing that $\mu(N) \alpha_{i, a t t}(N)=\mu_{0} \alpha_{(i, a t t) 0}$ does not depend on $N$. Therefore one can write $v_{i, a t t}=N f_{i, a t t}(|E| / N)$; this means that the number of impact ionization or attachment events produced by a given electron density at a given reduced electric field is proportional to the number density of air molecules.

We assume that initially the atmosphere is electrically neutral but with a pre-ionization $n_{e}(z)=$ $n_{+}(z)=n_{e 0} \exp (z / \ell)$ where $\ell=2.86 \mathrm{~km}$ and $n_{e 0}=7.6 \cdot 10^{-14} \mathrm{~cm}^{-3}$ [30]. This profile is based on night-time observation in conditions of normal weather. Figure 4 shows a comparison of this profile with others in the literature. Note that due to radioactivity, the actual ionization at ground level is much higher that $n_{e 0}$ and our expression is only valid at mesospheric altitudes.

The charged thunderstorm cloud that induces the sprite discharge is simulated by a point charge located at $L_{Q}=10 \mathrm{~km}$ above ground that increases linearly in time due to a constant cloud-earth current of $J=30 \mathrm{kA}$. The current moment change is hence $J L_{Q}=300 \mathrm{kAkm}$ and the charge moment change at time $t$ is $C M C=J L_{Q} t$. This is a simplified model of the current moment waveform for a short-delayed sprite reported in [24].

To solve the equations numerically we assume cylindrical symmetry around the vertical axis through the cloud charge; and we solve the Poisson equation between ground level and $85 \mathrm{~km}$, assuming that the earth is a perfect conductor and that above $85 \mathrm{~km}$ the time response of the 


\section{SUPPLEMENTARY INFORMATION}

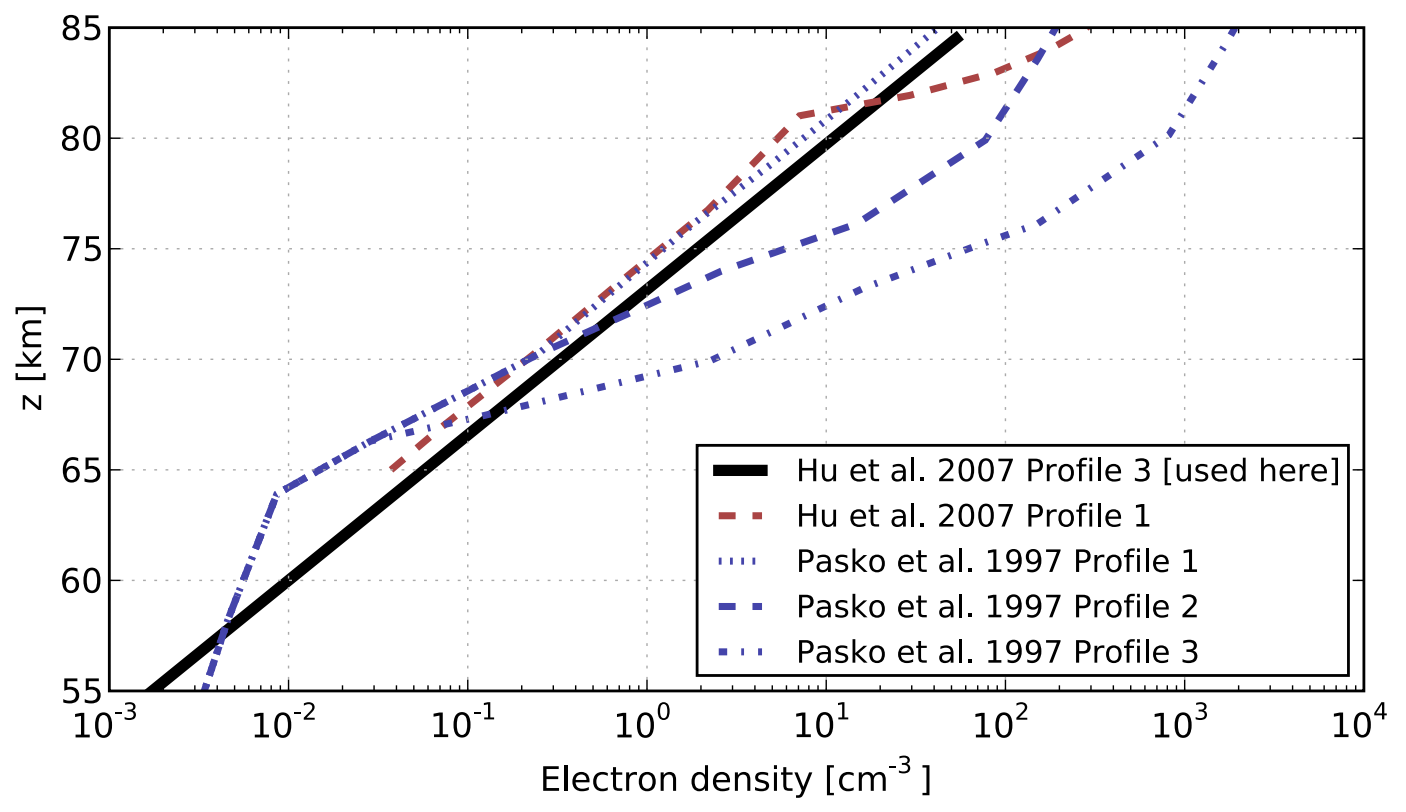

FIG. 4: Initial electron density used in our simulation (solid, thick line) and some other profiles found in the literature. Hu et al. refers to [25]; Pasko et al. refers to [17].

ionosphere, shorter than $0.2 \mathrm{~ms}$ is fast enough compared to our typical times to replace it by a perfect conductor as well. To make computations faster, we restrict the solution of the density equations to a layer between $55 \mathrm{~km}$ and $85 \mathrm{~km}$. Both for the densities and for the Poisson equation, the domain extends up to a radius of $20 \mathrm{~km}$ in the lateral direction, where homogeneous Neumann boundary conditions (that create symmetry planes) are imposed for the particle densities.

The electric field is calculated in two parts: the cloud charge is represented by a point charge and conducting earth and ionosphere above $85 \mathrm{~km}$ altitude are represented by a sequence of 2 mirror charges on each side. Then we add the fields created by the charges of sprite halo and sprite streamer, obtained by solving the Poisson equation inside a cylinder that extends from ground level to $85 \mathrm{~km}$ and has a radius of $20 \mathrm{~km}$, using homogeneous Neumann boundary conditions in the lateral boundaries and homogeneous Dirichlet in the upper and lower boundaries.

We solve equations (1)-(6) in adaptively refined grids as described in [13,23] with a maximum grid size of $\Delta r_{\max }=\Delta z_{\max }=100 \mathrm{~m}$ and a minimum of $\Delta r_{\min }=\Delta z_{\min }=\Delta z_{\max } / 32 \approx 3 \mathrm{~m}$. We also tested our simulations on minimal grids of 4 and $2 \mathrm{~m}$. This did not change significantly the time of streamer emergence and the velocity and diameter of the sprites. Given also the relevant length scales discussed in the text and visible in the figures, we conclude that the numerical grid is fine 
enough to appropriately resolve the structures.

\section{Comparison with weakly non-linear models}

The model (1)-(6) can also be written in terms of the charge density $\rho=\mathrm{e}\left(n_{+}-n_{-}-n_{e}\right)$, the atmospheric conductivity $\sigma=\mathrm{e} \mu n_{e}$ and the ionic charge balance $\rho_{i}=\mathrm{e}\left(n_{+}-n_{-}\right)$, where we have dropped the dependence on the neutral gas density to simplify the notation. From (1)-(3) we trivially obtain a charge conservation equation

$$
\partial_{t} \rho=-\nabla \cdot \mathbf{j}
$$

where $\mathbf{j}=\sigma \mathbf{E}+\mathrm{e} D \nabla n_{e}$ is the electrical current. Subtracting (3) from (2) we also obtain

$$
\partial_{t} \rho_{i}=\left(v_{i}-v_{a}\right) \sigma / \mu+\mathrm{e} S_{p h}
$$

And if we multiply (1) with e $\mu$ we get

$$
\partial_{t} \sigma=\mu \nabla \cdot \mathbf{j}+\left(v_{i}-v_{a}\right) \sigma+\mathrm{e} \mu S_{p h}
$$

Many atmospheric electricity models $[17,25,26]$ implicitly assume that $\left(v_{i}-v_{a}\right) \sigma$ dominates over $\mu \nabla \cdot \mathbf{j}$ and $\mathrm{e} \mu S_{p h}$, thus reducing (8) and (9) to

$$
\begin{gathered}
\partial_{t} \rho_{i}=\left(v_{i}-v_{a}\right) \sigma / \mu, \\
\partial_{t} \sigma=\left(v_{i}-v_{a}\right) \sigma .
\end{gathered}
$$

We remark that

1. Models in $[17,25,26]$ include also the ionic conductivity $\sigma_{i}=\mathrm{e} \mu_{+} n_{+}+\mathrm{e} \mu_{-} n_{-}$, which we have neglected (see main text).

2. Equation (10) is decoupled from (7) and (11) and therefore it can be and is usually left out from atmospheric models.

The simplifications leading to (10) and (11) amount to neglecting photo-ionization and assuming that electron transport does not significantly alter the ambient conductivity, thus obtaining a weakly non-linear system of equations. This assumption is valid for smooth density profiles but breaks down when strong gradients are present. It must be stressed that streamers are a strongly non-linear process and the terms neglected in (11) play an essential role in their inception and propagation. 


\section{OPTICAL EMISSIONS}

To compare the outcome of our simulation with the optical observations of sprites, we implemented the model of sprite emission described by Liu and Pasko ${ }^{12}$. The model includes a localfield-dependent excitation by electron impact of the $B^{3} \Pi_{g}$ and $C^{3} \Pi_{u}$ states of $\mathrm{N}_{2}$ and the $B^{2} \Sigma_{u}^{+}$state of $\mathrm{N}_{2}^{+}$. If they are not quenched, these states relax to their ground state by emitting photons in the first and second positive bands of $\mathrm{N}_{2}$ and the first negative band of $\mathrm{N}_{2}^{+}$. We used the excitation, quenching and emission rates detailed in Table 1 of [12], corrected by altitude-dependent number densities of air molecules. In this paper we report only the emissions from the first positive band of $\mathrm{N}_{2}$, which is responsible for most of the intensity recorded in ground based observations.

The intensities recorded in direct observations of sprites correspond to the emissions from an integrated line-of-sight perpendicular to the plane of the camera. The finite opening time of the camera - or, in the case of [9], the phosphor persistence of the intensifier - is simulated in our case by averaging over a time of $0.25 \mathrm{~ms}$.

The result appears in Figure 2. The emissions from the emerging streamer head are approximately $1.2 \cdot 10^{9}$ Rayleigh (1 Rayleigh, abbreviated $1 \mathrm{R}$, is $10^{10}$ photons per second per square meter), in agreement with the estimations of [11].

In Figure 6c we show the optical emissions around the emerging streamer at time $4.42 \mathrm{~ms}$. From these optical emissions we can estimate a visible diameter of the streamer of approximately $600 \mathrm{~m}$.

\section{SIMULATED ELECTRIC FIELDS}

Our simulations are based on a classical gas discharge model, and therefore, of course, support the classical breakdown model for sprites: to initiate a sprite, the cloud charge must generate local electric field strengths $E$ above the breakdown threshold $E_{k}$. The occurrence of this necessary criterion is investigated in [25,26], but not whether subsequently a sprite is actually formed. The evolution of the reduced electric fields $E / E_{k}$ for the time steps of Figures 1 and 2 is represented in Figure 4. We recall that $E_{k}$ strongly depends on altitude and that in regions with $E / E_{k}>1$, the ionization grows while elsewhere it decreases.

A zoom into the emerging sprite streamer at time $t=4.42 \mathrm{~ms}$ is shown in Fig. 6 . The figure shows a typical positive streamer that propagates in the high-field region due to photo-ionization. 

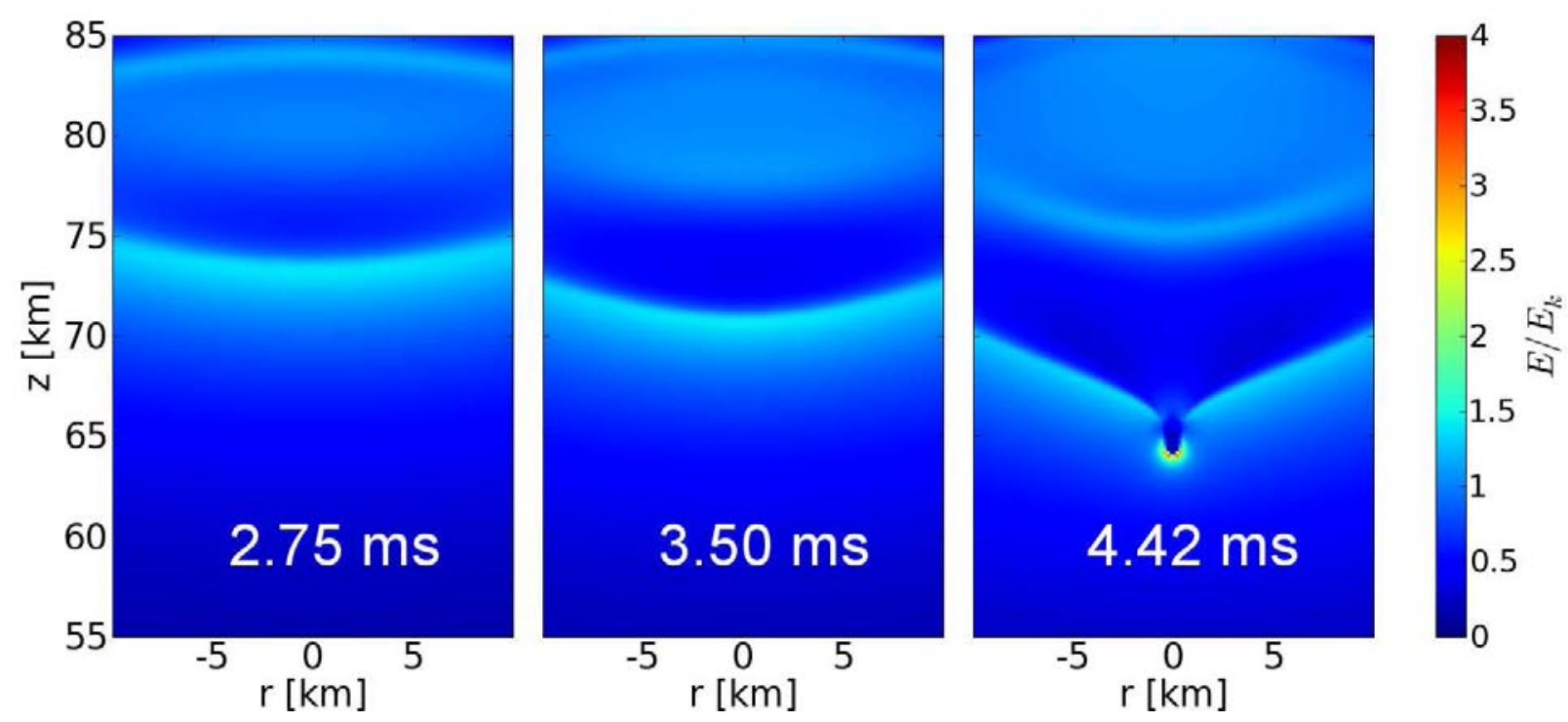

FIG. 5: Reduced electric fields corresponding to the simulation of Figures 1 and 2. Shown is here the reduced electric field strength $E / E_{k}$ where $E_{k}$ depends on density and therefore on altitude. The electron density grows due to ionization reactions where $E / E_{k}>1$.

The streamer emerges from the tip of the screening-ionization wave where the electric field is focused due to the curvature of the front. The field enhancement in the streamer head is about 4 times $E_{k}$.

At time $t=2.46 \mathrm{~ms}$ of Fig. 2 in Ref. [9], the single initial sprite streamer breaks up into many channels. The same happens in our simulation as well after about $2 \mathrm{~km}$ of propagation. However, as we have implemented cylindrical symmetry around the cloud charge axis to reduce computational complexity, the evolution is not physical anymore after branching and therefore not shown.

\section{HORIZONTAL INHOMOGENEITIES}

In our reported simulations the initial electron density depends only on the altitude and is thus uniform in the horizontal direction. However, many atmospheric processes such as meteor trails, gravity waves and the previous electrical activity of the thunderstorm may create horizontal inhomogeneities.

Presently our model is limited to cylindrically symmetrical configurations and we could only introduce inhomogeneities with cylindrical symmetry. In particular, we tested the effect of pertur- 

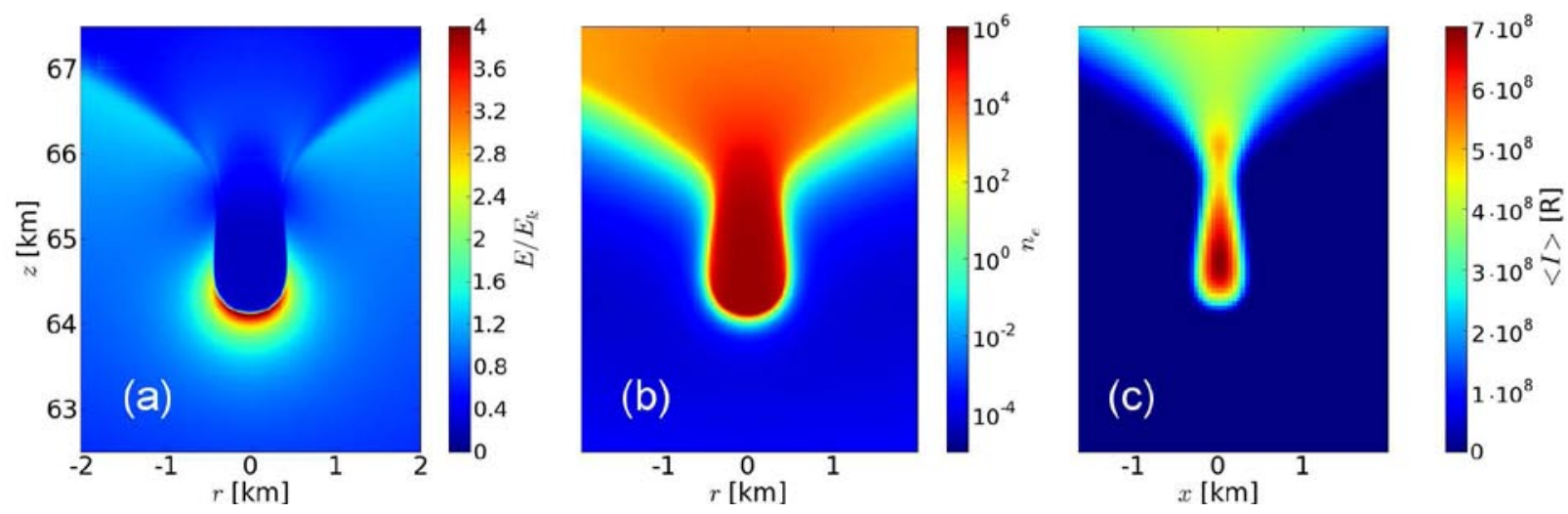

FIG. 6: Zoom into the last time step $t=4.42 \mathrm{~ms}$ of Figures 1, 2 and 4 showing (a) the reduced field, (b) the electron density and (c) the optical emissions averaged over the previous $0.25 \mathrm{~ms}$.

bations to the initial density of the form

$$
n_{e}^{\prime}(r, z)=n_{e}(z)\left(1+\epsilon e^{-r^{2} / R^{2}}\right)
$$

where $n_{e}(z)$ is the unperturbed electron density (plotted in Fig. 4), $\epsilon$ measures the amplitude of the perturbation and $R$ determines its width.

When we run a simulation using $\epsilon=0.2$ (i.e., the maximum increase of electron densities is $20 \%$ ) and $R=500 \mathrm{~m}$, we found that sprites emerge after $3.30 \mathrm{~ms}$ at $71 \mathrm{~km}$ altitude and for a charge moment change of about $1000 \mathrm{C} \mathrm{km}$. The emerging sprite has an optical diameter of about $250 \mathrm{~m}$ and propagates at $\sim 2.5 \cdot 10^{7} \mathrm{~m} / \mathrm{s}$. The calculated optical emissions from this sprite are shown in Figure 7: note that in this case the sprite looks dimmer due to the lower charge moment change.

The probable reason is that the horizontal inhomogeneity triggers the instability of the screening-ionization wave much earlier. This effect could explain the observation of sprites produced by flashes with very low charge moment changes that do not generate sprites in a horizontally homogeneous model.

\section{LATERAL EXTENSION}

Computer memory limited the spatial extension of the simulation. Adaptive refinement allowed us to use a high resolution only in the interesting areas but we used relatively high resolutions in the complete leading edge of the wide ionization front to avoid matching problems between different levels of resolution. The result is that we run out of memory when we tried to simulate domains 

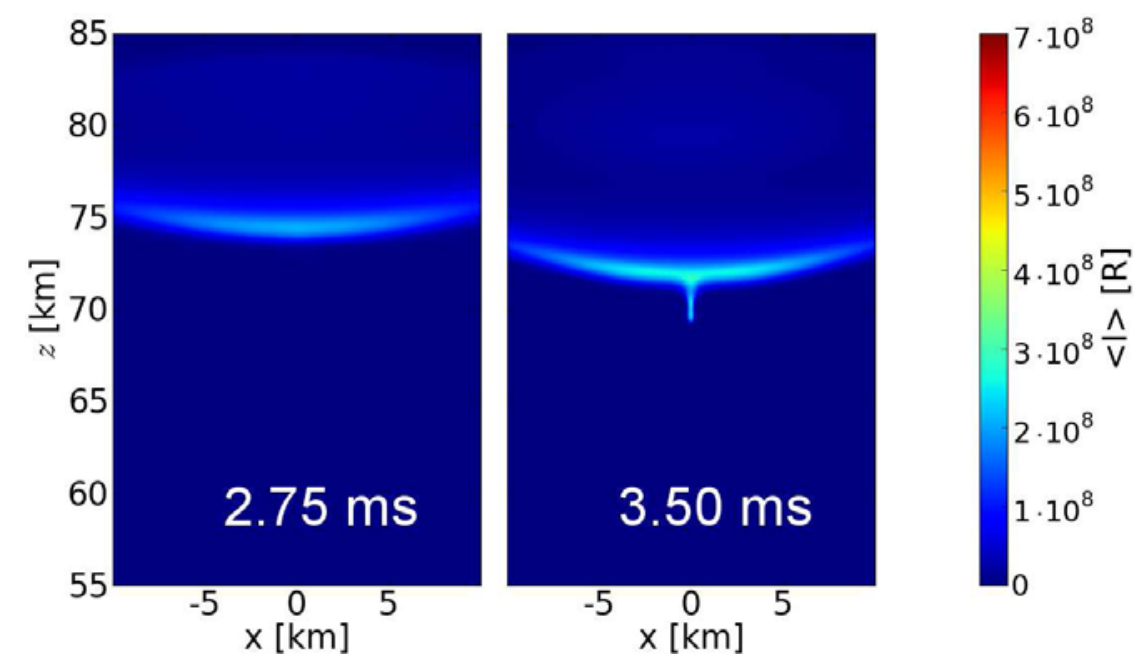

FIG. 7: Optical emissions of a simulation where the initial electron density was perturbed by a horizontal inhomogeneity (see text). We have used the same color scale as in Figure 2 to show that in this case the streamer looks much dimmer. Note that in this case the sprite emerges above $70 \mathrm{~km}$ altitude, in closer agreement with observations.

wider than about $20 \mathrm{~km}$. However, since high resolution is not needed in the initial halo stage, we can compare our results with those obtained in a much wider domain, but with a low resolution ( $\Delta r=\Delta z=150 \mathrm{~m})$ everywhere. The results are shown in Fig. 8. 


\section{SUPPLEMENTARY INFORMATION}
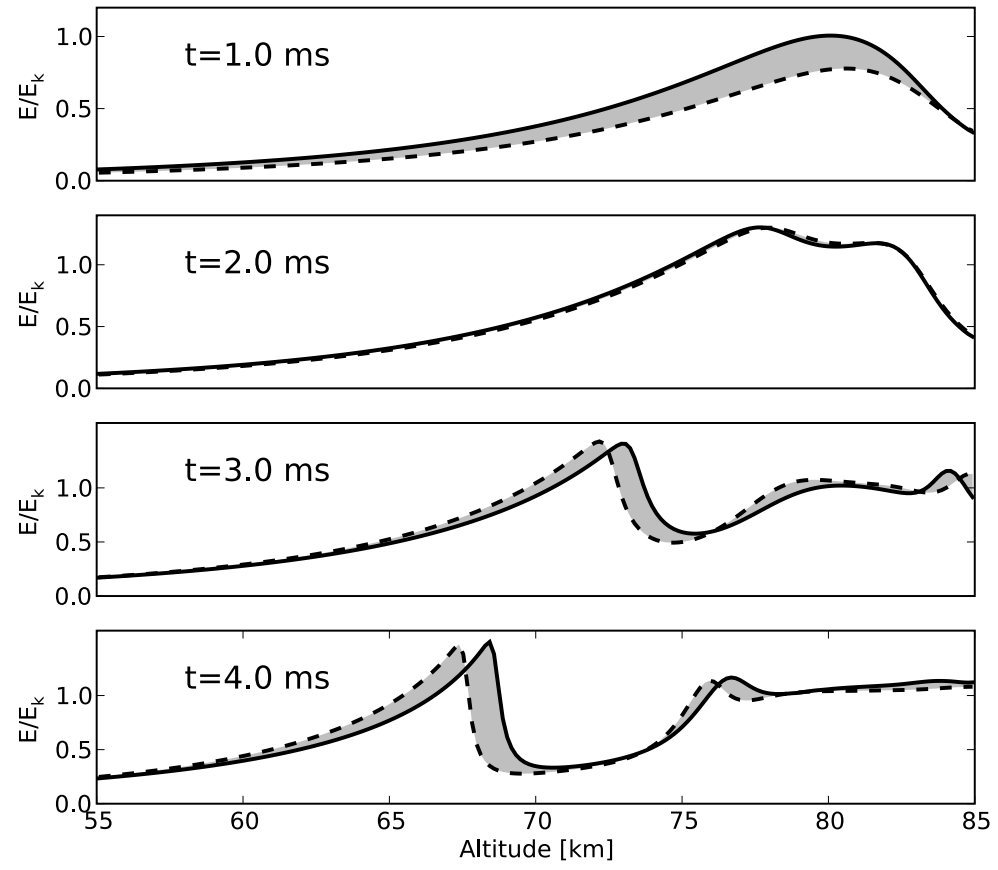

FIG. 8: Comparison between the reduced electric field from a simulation with a maximum radial extension of $20 \mathrm{~km}$ (as used in the main text; solid line) and one with $80 \mathrm{~km}$ (dashed line). Although there is some difference, the qualitative behavior of both simulations is remarkably close. In particular, the maximal field enhancement is similar. 